\title{
In Search of Conversational Grain Size: Modelling Semantic Structure Using Moving Stanza Windows
}

\author{
Amanda L. Siebert-Evenstone \\ Wisconsin Center for Education Research \\ University of Wisconsin - Madison, United States \\ alevenstone@wisc.edu \\ Golnaz Arastoopour Irgens \\ Wisconsin Center for Education Research \\ University of Wisconsin - Madison, United States \\ Wesley Collier \\ Wisconsin Center for Education Research \\ University of Wisconsin - Madison, United States \\ Zachari Swiecki \\ Wisconsin Center for Education Research \\ University of Wisconsin - Madison, United States \\ Andrew R. Ruis \\ Wisconsin Center for Education Research \\ University of Wisconsin - Madison, United States \\ David Williamson Shaffer \\ Wisconsin Center for Education Research \\ University of Wisconsin - Madison, United States
}

\begin{abstract}
Analyses of learning based on student discourse need to account not only for the content of the utterances but also for the ways in which students make connections across turns of talk. This requires segmentation of discourse data to define when connections are likely to be meaningful. In this paper, we present an approach to segmenting data for the purposes of modelling connections in discourse using epistemic network analysis. Specifically, we use epistemic network analysis to model connections in student discourse using a temporal segmentation method adapted from recent work in the learning sciences. We compare the results of this study to a purely conversation-based segmentation method to examine the affordances of temporal segmentation for modelling connections in discourse.
\end{abstract}

Keywords: Sliding window, epistemic network analysis, segmentation, discourse analysis 
(2017). In search of conversational grain size: Modelling semantic structure using moving stanza windows. Journal of Learning Analytics, 4(3), 123-139. http://dx.doi.org/10.18608/jla.2017.43.7

\section{NOTES FOR PRACTICE}

- When analyzing learning based on student discourse we need to account not only for the content of student talk but also the ways in which students make connections within a conversation. However, this requires segmentation of discourse data to define when connections are likely to be meaningful.

- This methods paper uses Epistemic Network Analysis to understand how connections are modeled based on the conversation method, which models connections within an entire activity, and the moving stanza window method, which models connections within a conversation by dividing the activity into multiple overlapping stanzas

- An important benefit of the moving stanza window method is that it models the role of individual contributions to group discussions. By using a sliding window of fixed size to establish the analytic context, researchers can create models of discourse that update with each new contribution to the conversation.

- Many CSCL environments already include integrated feedback and assessment; however, the ability to use the moving stanza window method to model individual contributions to group discussions in a chat's recent temporal context would allow teachers the ability to assess real-time student performance in online environments.

\section{INTRODUCTION}

Analyzing high-volume discourse data is a challenge in computer-supported collaborative learning (CSCL) environments because student conversations in such environments are characterized not only by what is said but by patterns of language use within social practices (Gee, 1990). This suggests that analyses of learning based on student discourse need to account not only for the content of the utterances but also for the ways in which students make connections across turns of talk. Any analysis of such connections, however, requires segmentation of discourse data to identify the conditions under which connections are likely to be meaningful (Hearst, 1994). In this paper, we present an approach to segmenting data for the purposes of modelling connections in discourse. Specifically, we use epistemic network analysis (Shaffer et al., 2009) to model connections in student discourse using a temporal segmentation method adapted from recent work in the learning sciences (Dyke, Kumar, Ai, \& Rosé, 2012; Suthers \& Desiato, 2012). We compare the results to a conversation-based segmentation method to examine the affordances of temporal segmentation for modelling connections in discourse.

\section{THEORY}

There are a number of theoretical perspectives in the learning sciences that describe one's understanding of a topic, process, domain, or practice in terms of the structure of understanding; that is, the way concepts, skills, and habits of mind are related to one another systematically. Chi, Feltovich, and Glaser (1981), for example, found that experts in physics organize their understanding differently than novices. Bransford, Brown, and Cocking (1999) showed that the organization of experts' content knowledge reflects their deep understanding of subject matter. DiSessa (1988) suggests that that while solving physics problems requires understanding basic concepts from the discipline, deep and 
(2017). In search of conversational grain size: Modelling semantic structure using moving stanza windows. Journal of Learning Analytics, 4(3), 123-139. http://dx.doi.org/10.18608/jla.2017.43.7

systematic understanding comes from linking such concepts to one another within a theoretical framework. Similarly, Shaffer (2012) characterizes learning as the development of an epistemic frame: a pattern of associations among knowledge, skills, habits of mind, and other cognitive elements that characterizes communities of practice, or groups of people who share similar ways of framing, investigating, and solving complex problems.

Not surprisingly, research on discourse processing suggests that connections among concepts are made primarily on a topic-by-topic basis rather than across discourse as a whole. For example, Gernsbacher's (1991; see also Graesser, Gernsbacher, \& Goldman, 1997) theory of language processing suggests that students use hierarchical organization of content to build understanding. Discourse is structured by topic, with concepts having clear relationships to one another within topics and few relationships across topics.

Similarly, epistemic network analysis (ENA) analyzes the structure of connections in student discourse by looking at the co-occurrence of concepts within the conversations, topics, or activities that take place during learning. Building on the idea of learning as the development of an epistemic frame, ENA creates a discourse network model of thinking by identifying the co-occurrence of skills, knowledge, values, and other elements of work in a particular community of practice (Shaffer et al., 2009). The co-occurrences are identified within collections of related utterances, which are nested within activities, a fundamental unit of analysis in ENA. Prior work by Collier, Ruis, and Shaffer (2016) has shown that analyzing connections within activities is a more sensitive measure than analyzing correlations of ideas in a corpus of data overall, and a number of studies (Arastoopour, Swiecki, Chesler, \& Shaffer, 2015; Chesler et al., 2015; Knight, Arastoopour, Shaffer, Shum, \& Littleton, 2014) have used ENA to analyze student learning at the activity level.

There are, however, two problems with such an approach. First, as Stahl, Koschmann, and Suthers (2006) argue, learning needs to be analyzed at both the group and the individual level. Stahl (2009), for example, conducted parallel qualitative analyses of the mathematics learning of a group and of the individuals in the group. But as Cress and Hesse (2013) point out, because learners work in groups, simple t-tests and ANOVAs do not effectively model the influence that groupmates have on one another. Thus, creating a quantitative model of group discourse that accounts for the contributions of any single individual within the group discussion remains a challenge.

A second problem is that the aggregation of connections using the entire activity may incorrectly connect ideas that are in fact not within the same context (Arvaja, Salovaara, Häkkinen, \& Järvelä, 2007). While ideas are surely connected within conversations or activities, such connected ideas are most likely to occur in close temporal proximity. During discussions, students simultaneously build group and individual understanding by "saying" and replying to "what is said" (Wells, 1999). Speech typically addresses another instance of speech and anticipates a response (Bakhtin, 1986). Because "thinking and speech are, in this sense, always derivative of prior thinking and speech" (Smagorinsky, 2011, p. 23), students build on the ideas of their team members to mediate their discussion of concepts. Therefore, 
(2017). In search of conversational grain size: Modelling semantic structure using moving stanza windows. Journal of Learning Analytics, 4(3), 123-139. http://dx.doi.org/10.18608/jla.2017.43.7

to measure connections in conversations, we need a method to model connection-making on shorter time scales than entire activities.

Recent work by Dyke and colleagues (2012) and Suthers and Desiato (2012) proposes using sliding window analyses to model temporal connections in discourse within their recent temporal context. Rather than creating summary values for all utterances in an activity, a sliding window can analyze recent temporal context by computing a value for a smaller section of an activity - typically a small amount of time (e.g., 10 seconds) or a small number of utterances (e.g., three turns of talk; Dyke et al., 2012). The window is sliding in the sense that a summary value is computed for each utterance, based on the preceding lines of talk (e.g., the preceding 10 seconds or three lines of talk). Other forms of sliding window analyses have been used to identify shifts in topic (Rosé et al., 2008), visualize semantic similarities between utterances (e.g., PolyCAFe; Trausan-Matu, Dascalu, \& Rebedea, 2014), and more generally to provide new insights on previously analyzed data (Dyke et al., 2012). By analyzing discourse in smaller segments that are temporally related, a sliding window approach is less likely to take an utterance out of context than an approach that examines connections across an entire activity.

Although sliding windows measure discourse on small time scales, sliding windows alone do not measure connections among codes nor do they address how people collaboratively co-construct knowledge. To measure connections between ideas, Suthers and Desiato (2012) proposed measuring uptake - modelling structures of connections that show when participants refer to prior events and how such references help continue conversation. However, while Suthers and Desiato's model showed when each actor used another actor's contribution, this model only showed whether a connection was made, not what connection was made nor the semantic structure of connections.

In what follows, we model the semantic structure of connections in discourse and use ideas from Shaffer (2017) that build on Gee's (1990) work to create an ENA model using a moving window approach. When analyzing discourse, first we identify the smallest unit of analysis as a single line, which in CSCL discourse is often a turn of talk. After designating lines, we group these lines together into conversations, which are the set of all lines from a single team during a single activity. For instance, all chat utterances in a $\mathrm{CSCL}$ environment may be designated as a line and then grouped by each activity in that environment into a conversation. By segmenting data into a conversation, we assume that all lines within that conversation are equally related, when they may not be. Therefore, within conversations we can define stanzas, which are a set of related lines within that conversation. Gee argues that single lines or utterances in talk are grouped together into sets of related lines called stanzas. The analogy is to stanzas in a poem, in which lines are related within stanzas, and within a poem, which could be considered a conversation, but not across poems. Using this idea, ENA can model the co-occurrence of ideas by conversations or by stanzas within conversations.

In this study, we use the idea of conversations and stanzas to delineate two different approaches to modelling connections using ENA. In both cases, ENA models connections among concepts: 1) by 
(2017). In search of conversational grain size: Modelling semantic structure using moving stanza windows. Journal of Learning Analytics, 4(3), 123-139. http://dx.doi.org/10.18608/jla.2017.43.7

identifying a conversation as an entire activity; and 2) by identifying stanzas as collections of utterances within conversations. Specifically, they are as follows:

1. The Conversation ${ }^{1}$ Method models connections within an entire activity; that is, all the utterances within an activity are related to one another. Or, equivalently, each activity is composed of a single stanza.

2. The Moving Stanza Window Method models connections within a conversation by dividing the activity into multiple overlapping stanzas; that is, utterances are related to one another only within some designated stanza window. Thus, the moving stanza window method models connections only when utterances are in close temporal proximity within an activity.

In what follows, we compare the two ENA segmentation methods by looking at data from a CSCL learning environment in which students collaboratively design solutions to engineering problems. To evaluate the strengths and limitations of the two approaches to segmentation, we created ENA models using both the conversation method and the moving stanza window method. In this study, we focus on the discourse of one representative team and ask:

Does the moving stanza window method provide information about group discourse that the conversation method does not?

\section{METHODS}

\subsection{The Engineering Virtual Internship RescuShell}

RescuShell is a 10-week long engineering virtual internship, in which students roleplay as engineering interns at a fictional mechanical engineering design firm working to develop robotic legs for a mechanical exoskeleton for use by rescue personnel. Students use an online work portal with email and an instant messaging chat window to engage in 17 different activities that simulate various steps in the design process, including reviewing and summarizing research reports, creating device prototypes, discussing design choices with teammates, and working to balance the needs of various internal consultants and external clients. During these activities, students research how each of the five internal consultants in RescuShell prioritize two performance parameters and request specific threshold values for each of these parameters. For example, the biomedical engineer prefers a device with high agility and high safety, while the environmental engineer prefers a device with a high recharge interval and a low cost. Students try to meet the internal consultants' requests by exploring how various technical constraints (e.g., actuators, powers sources, range of motion, sensors, and materials) affect the performance parameters. However, each of the internal consultant's concerns are in conflict with one another (e.g., as recharge interval decreases, cost also increases). Therefore, students must balance

\footnotetext{
${ }^{1}$ In other writings, we have referred to the conversation method as the strophe or topic method. For this analysis, we have simplified the language to conversation to reflect that we separated the discourse based on entire conversations about a topic or activity.
} 
(2017). In search of conversational grain size: Modelling semantic structure using moving stanza windows. Journal of Learning Analytics, 4(3), 123-139. http://dx.doi.org/10.18608/jla.2017.43.7

client and consultant requests and justify their design decisions when designing and testing exoskeleton prototypes.

In this study, we focused on the first eleven activities of the internship, during which students were randomly assigned to one of five teams, each of which explored the use of a particular actuator in the exoskeleton design (hydraulic, PAM, electric, pneumatic, or series elastic). Forty-four first-year engineering students participated in the virtual internship, which took approximately 15 hours to complete. From this sample, we selected one representative team from the broader sample and analyzed how these five students ( 4 male, 1 female) discussed the design problem in the first half of the internship.

\subsection{Discourse Analyses}

\subsubsection{Coding student chats}

We collected chat log data from teams and segmented by utterance, defined as when a student sent a single message in the chat program. We developed a set of codes to represent the key elements the engineering design process (see Table 1).

Table 1. Engineering Design Coding Scheme

\begin{tabular}{|c|c|c|}
\hline Code Name & Description & Example \\
\hline $\begin{array}{l}\text { Design } \\
\text { Reasoning }\end{array}$ & $\begin{array}{l}\text { Referring to design development, } \\
\text { prioritization, trade-offs, and } \\
\text { design decisions }\end{array}$ & $\begin{array}{l}\text { "Aluminum and Composite are good } \\
\text { options. Steel can carry a big load, but it is } \\
\text { heavy and weighs down on the recharge } \\
\text { interval, and it is a costly option." }\end{array}$ \\
\hline $\begin{array}{l}\text { Performance } \\
\text { Parameters }\end{array}$ & $\begin{array}{l}\text { Referring to attributes: payload, } \\
\text { recharge interval, agility, safety, } \\
\text { or cost. }\end{array}$ & $\begin{array}{l}\text { "My device has a pretty good safety, } \\
\text { payload, agility, and recharge interval; the } \\
\text { cost is a little high though." }\end{array}$ \\
\hline Technical & Referring to inputs: actuators, & "Our two best were both made with \\
\hline Constraints & $\begin{array}{l}\text { ROM, materials, power sources, } \\
\text { or sensors. }\end{array}$ & $\begin{array}{l}\text { Aluminum, NiCd Batteries, Piezoelectric } \\
\text { sensors, and Pneumatic actuators." }\end{array}$ \\
\hline $\begin{array}{l}\text { Client and } \\
\text { Consultant } \\
\text { Requests }\end{array}$ & $\begin{array}{l}\text { Referring to or justifying } \\
\text { decisions based on internal } \\
\text { consultant's requests or client's } \\
\text { health or comfort }\end{array}$ & $\begin{array}{l}\text { "We tried to meet at least the minimum of } \\
\text { each of the internal consultant's requests." }\end{array}$ \\
\hline Collaboration & $\begin{array}{l}\text { Facilitating a joint meeting or the } \\
\text { production of team design } \\
\text { products. }\end{array}$ & "How should we make our team batch?" \\
\hline Data & $\begin{array}{l}\text { Referring to or justifying } \\
\text { decisions based on numerical } \\
\text { values, results tables, graphs, } \\
\text { research papers, or relative } \\
\text { quantities. }\end{array}$ & $\begin{array}{l}\text { "I thought that safety near the maximum } \\
\text { was not very good (close to } 225 \text { - one had } \\
218 \text { RPN), but other than that, I was fine } \\
\text { with the safety as long as it was around } 200 \\
\text { or lower." }\end{array}$ \\
\hline
\end{tabular}


(2017). In search of conversational grain size: Modelling semantic structure using moving stanza windows. Journal of Learning Analytics, 4(3), 123-139. http://dx.doi.org/10.18608/jla.2017.43.7

Because the chat data had a high volume of data (3824 utterances), we applied the coding scheme to each utterance using an automated coding process that uses keywords for regular expression matching (Shaffer et al., 2015; Arastoopour et al., 2015). We validated all six codes using a series of comparisons between two human raters and the computer with resulting Cohen's kappa scores between 0.83 and 1.00 (see Table 2). The interrater reliability analysis shows that all pairwise agreements among rater 1 , rater 2, and the computer meet standards for kappa (Landis \& Koch, 1977). We used a Monte Carlo rejection technique, Shaffer's rho, to determine for each kappa value the likelihood that it would be found by two coders if their the true rate of agreement was less than kappa of 0.65 (Shaffer et al., 2015). As shown in Table 2 below, all of the kappa values achieved have Shaffer's rho values less than 0.05 , meaning that the Type I error rate for assuming that if the coders were to code the whole data set they would have a level of agreement over kappa of 0.65 .

Table 2. Interrater Reliability Analysis between Two Raters and an Automated Coding Scheme

\begin{tabular}{llll}
\hline Code Name & $\begin{array}{l}\text { Kappa between } \\
\text { Rater } \mathbf{1} \text { and } \\
\text { Rater 2 }\end{array}$ & $\begin{array}{l}\text { Kappa between } \\
\text { Rater 1 and } \\
\text { AutoCoder }\end{array}$ & $\begin{array}{l}\text { Kappa between } \\
\text { Rater } \mathbf{2} \text { and } \\
\text { AutoCoder }\end{array}$ \\
\hline Design Reasoning & $0.89^{* *}$ & $0.89^{*}$ & $0.89^{* *}$ \\
Performance Parameters & $0.89^{* *}$ & $1.00^{* *}$ & $0.89^{* *}$ \\
Technical Constraints & $0.83^{* *}$ & $0.94^{* *}$ & $0.89^{* *}$ \\
Client and Consultant & $1.00^{* *}$ & $1.00^{*}$ & $1.00^{* *}$ \\
Requests & & & \\
Collaboration & $1.00^{* *}$ & $1.00^{*}$ & $1.00^{* *}$ \\
Data & $0.9 * *$ & $0.87^{* *}$ & $0.89^{* *}$ \\
\hline
\end{tabular}

Note: ${ }^{*}$ rho $<0.05, * *$ rho $<0.01$

We then performed a chronologically oriented representations of discourse and tool-related activity (CORDTRA) analysis (Hmelo-Silver, Liu, \& Jordan, 2009) during one activity to show the temporal pattern of the six codes in student discourse. Researchers use CORDTRA diagrams as a visualization technique to reveal patterns in collaborative discourse. In a CORDTRA diagram, each horizontal line represents a code, each point on these lines represents an instance of a specific code, and the $X$-axis represents discourse units over time.

\subsubsection{Epistemic network analysis}

ENA models the structure of connections among engineering epistemic frame elements by quantifying the co-occurrences of codes within a stanza (Shaffer et al., 2009; Shaffer 2014). After defining the segmentation structure, ENA creates an adjacency matrix representing the co-occurrences of codes in each stanza. To construct an adjacency matrix, ENA assigns a one for each unique pair of codes that cooccur one or more times in those utterances, and a zero for each unique pair that does not co-occur in the stanza. ENA sums the adjacency matrices into a cumulative adjacency matrix, where each cell represents the number of stanzas (i.e., the number of adjacency matrices) in which that unique pair of codes was present. Each person's or team's collection of co-occurrences is thus represented by a cumulative adjacency matrix that summarizes the pattern of connections among codes. 
(2017). In search of conversational grain size: Modelling semantic structure using moving stanza windows. Journal of Learning Analytics, 4(3), 123-139. http://dx.doi.org/10.18608/jla.2017.43.7

ENA then converts the cumulative adjacency matrices into cumulative adjacency vectors that are projected into a high-dimensional space based on the co-occurrence of codes across segments. These cumulative adjacency vectors are normalized to control for the varying lengths of vectors by dividing each vector by its length; the resulting vector thus represents the relative frequency of co-occurrences. ENA then performs a singular value decomposition on the normalized vectors. This produces a rotation of the original high-dimensional space, such that the rotated space provides a reduced number of dimensions that capture the maximum variance in the data.

The resulting models can be visualized as networks in which the nodes in the model are the codes and the lines connecting the nodes represent the co-occurrence of two codes. Thus, we can quantify and visualize the structure of connections among engineering design codes, making it possible to characterize student discourse during the virtual internship.

\subsection{Comparison of Segmentation Procedures}

In this study, we compared two methods of segmenting data for use in ENA: the conversation method and the moving stanza window method. For the conversation segmentation method, ENA created one adjacency matrix for each activity and then summed the matrices across the 11 activities for a given team.

The moving stanza window method created a referent adjacency matrix for each utterance, known as the referring utterance. The referent adjacency matrix for each utterance was constructed from two types of co-occurrences of codes: 1) co-occurrences within the referring utterance, and 2) cooccurrences between the referring utterance and a specific number of previous utterances, known as the window. The moving window then moved to the next referring utterance and created the next referent adjacency matrix. This process continued until the end of the defined conversation and then ENA summed the matrices across all utterances for that unit. No windows were made across activities (conversations), only within them. Figure 1 shows how the conversation method and the moving stanza window method created different models of connectivity.

\begin{tabular}{|l|} 
Coded Data \\
\begin{tabular}{|l|}
\hline I slightly prioritized agility, in order to meet \\
Shawn Edwards' recommendations.
\end{tabular} \\
\hline $\begin{array}{l}\text { I chose light materials because I believe they } \\
\text { would like something comfortable to wear }\end{array}$ \\
\hline Yeah I agree \\
\hline $\begin{array}{l}\text { I was reviewing Paulo Henriquez's requests and } \\
\text { he was most focused on safety. }\end{array}$ \\
\hline But recharge interval may be irritating iftoo low \\
\hline Rescue workers are concerned about safety \\
\hline
\end{tabular}

(a)
Moving Stanza Window Method

\begin{tabular}{|l|}
\hline \multicolumn{2}{|l|}{ Design reasoning } \\
\hline \\
\hline Consultant request \\
\hline Parameter \\
\hline \multicolumn{1}{|l|}{ Data } \\
\hline
\end{tabular}

(b)

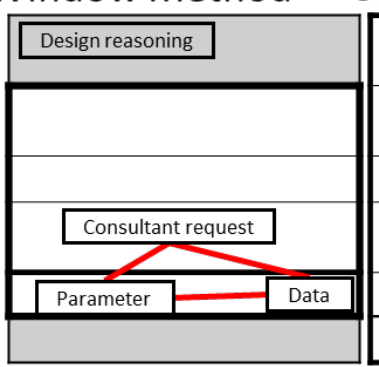

(c)

\section{Conversation Method}

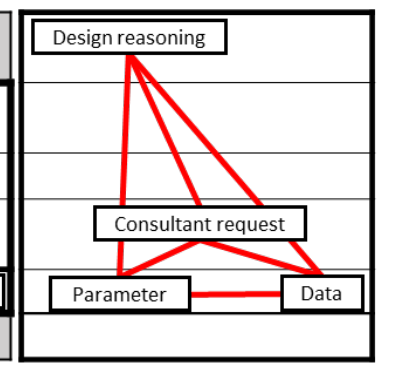

(d)

Figure 1. Example of coded data from one activity (a). The moving stanza window method analyzes connections within the referring utterance and between the referring utterance and the window (b). After analyzing a window, the moving stanza method slides to the next utterance and repeats the process of finding connections within and between the referring utterance and the window (c). The conversation method analyzes all connections in an activity (d). 
(2017). In search of conversational grain size: Modelling semantic structure using moving stanza windows. Journal of Learning Analytics, 4(3), 123-139. http://dx.doi.org/10.18608/jla.2017.43.7

Co-occurrences of codes within or across non-referring utterances were not included in the referent adjacency matrix, which eliminated double-counting of connections when the cumulative adjacency matrix was computed.

\subsection{Comparison of Network Models}

To analyze the different segmentation methods using ENA, we created three models: 1) a conversation model for all teams in the sample, 2) a moving stanza window model with a window size of three for all teams in the sample, and 3) a moving stanza window model with a window size of three for all students in the sample, based on a qualitative analysis of the data that suggested most explicit connections between ideas in the discourse occurred within a span of 4 or fewer lines (the referring utterance plus the preceding three turns of talk). All three of these sets were projected into the dimensional reduction for the team moving stanza model so the resulting networks could be compared. To analyze the differences between the two segmentation methods, we chose a representative team and closely examined the discourse of one team. First, we examined the team's discourse and compared the conversation model with the moving stanza window model, then we examined individual contributions to the team's discourse and used a moving stanza window model.

\section{$4 \quad$ RESULTS}

For the purposes of this analysis, we examined the conversations of one representative student project team. The Hydraulic team had five team members: Arden, Connor, Margaret, Jimmy, and Jordan. We modelled their collaborative design work over the first 11 activities of the virtual internship, which included background research into principles of biomechanics, as well as the design, testing, and evaluation of an initial prototype for a robotic exoskeleton.

\subsection{Conversation and Moving Stanza Window Models for the Hydraulic Team}

We used both the conversation method and the moving stanza window method to model the discourse of the team. Both models (see Figure 2) show that the connections to and between technical constraints and design reasoning were prominent in the group's design discussions. This is represented by larger node sizes and thicker lines in the ENA network graph linking the nodes that correspond to those discourse elements. This is, of course, hardly surprising, as the group's primary goal was to choose appropriate design features (input constraints) to maximize the function of their device.

However, the conversation method (Figure 2a) suggests that the Hydraulic team connected these features of design with explicit discussion of their collaboration process; in contrast, the moving stanza window method (Figure $2 \mathrm{~b}$ ) suggests that the team spent less time explicitly connecting talk about collaboration to their design work and more time linking the technical constraints and design reasoning to other elements of the problem space, representing explicit discussion about how to balance competing needs involved in the design process. 
(2017). In search of conversational grain size: Modelling semantic structure using moving stanza windows. Journal of Learning Analytics, 4(3), 123-139. http://dx.doi.org/10.18608/jla.2017.43.7

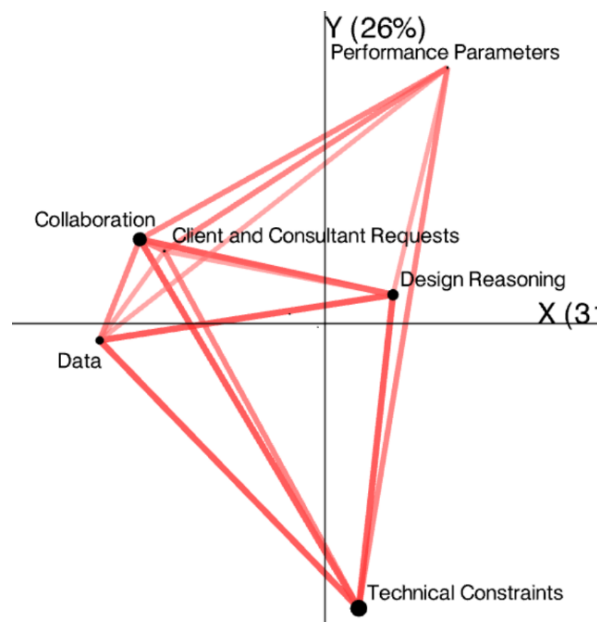

(a)

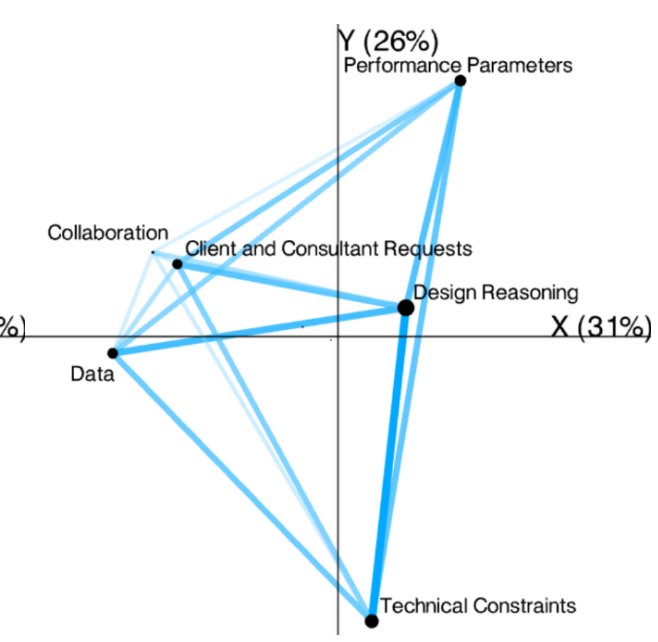

(b)

Figure 2. Network graphs of the Hydraulic team's discourse produced using (a) the conversation method and (b) the moving stanza window method. Thicker lines denote more frequent connections between codes. Percentages indicate the amount of variance explained by each dimension; in this analysis, $\mathbf{5 7 \%}$ of the total variance is accounted for in this data set.

This contrast is shown more clearly by computing the difference between the two network models (Figure 3). The difference between the network models is computed by subtracting the weight of each connection in one network from the corresponding weighted connection in the second network to obtain one network representation. Figure 3 shows a higher number of connections in the conversation method (red lines in the figure) to the node for collaboration, suggesting that links between the collaboration and other elements of the epistemic frame of engineering are a prominent feature of student discourse in this model. In contrast, the moving stanza window method (blue) suggests that students made more connections between the design elements of technical constraints, performance parameters, and design reasoning.

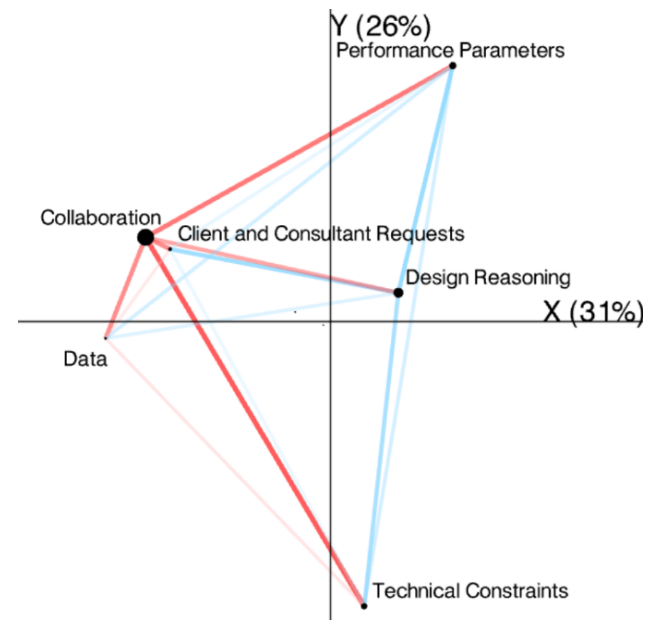

Figure 3. Subtracted network of the Hydraulic team's discourse, in which blue connections occur more frequently with the moving stanza method and red connections occur more frequently with the conversation method. 
(2017). In search of conversational grain size: Modelling semantic structure using moving stanza windows. Journal of Learning Analytics, 4(3), 123-139. http://dx.doi.org/10.18608/jla.2017.43.7

\subsection{Comparing Connections within a Single Conversation}

To further explore the differences between the two models of discourse, we examined the frequency of codes for each team within each conversation in the virtual internship. For example, when students met with their teammates to design devices, the discourse included references to the collaboration, which was one of the key differences between the two models. To understand why there was such a substantial difference in connections to collaboration, we examined patterns of code using a CORDTRA representation for this activity (Figure 4).

The CORDTRA shows that students explicitly talked about collaboration only at the start and at the end of the activity. In the previous analysis, applying the conversation method to this activity produced connections between collaboration and codes that appeared at any point within the activity, even though the CORDTRA revealed that students only talked explicitly about collaboration at the beginning and the end of the discussion.

In contrast, applying the moving stanza window produced connections between codes only if the codes co-occurred within recent temporal proximity; that is, within three utterances of the referring utterance. Thus, the moving stanza window model shows a less prominent role for collaboration.

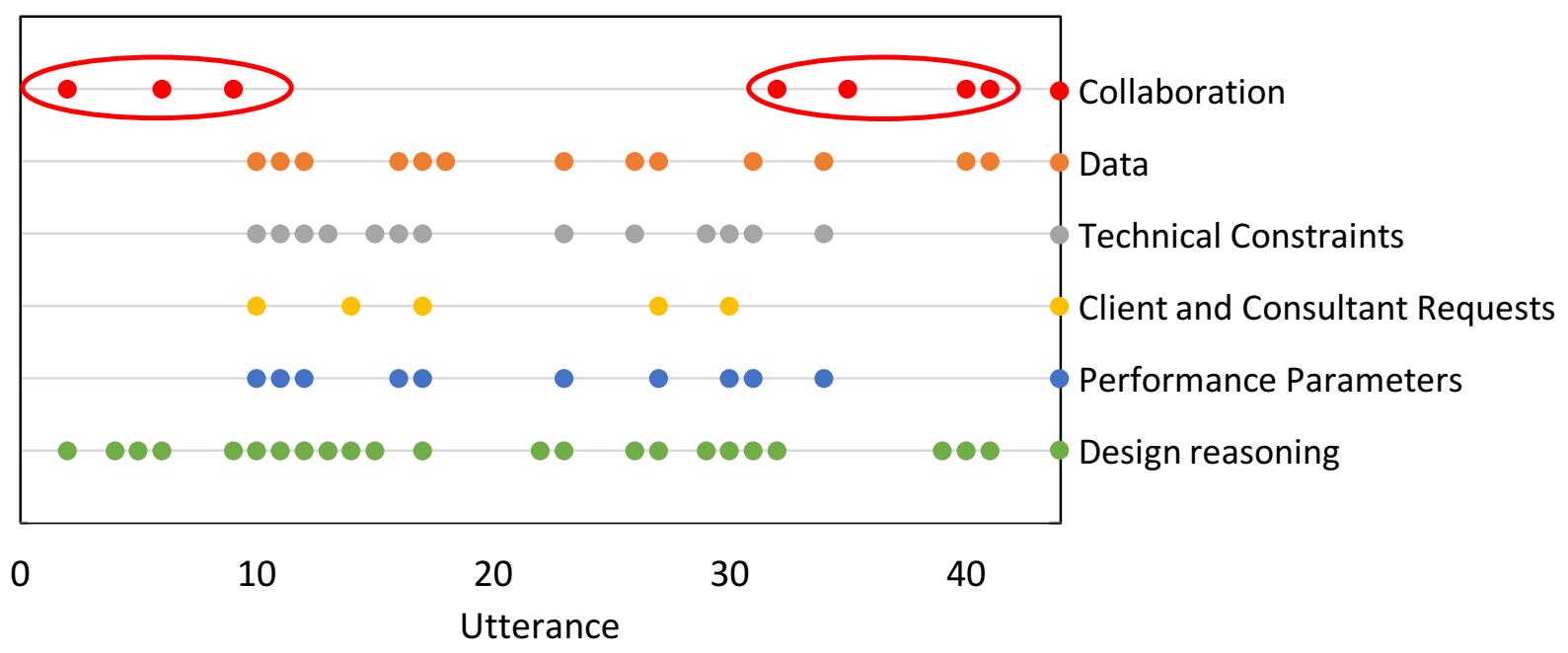

Figure 4. CORDTRA diagram of Hydraulic team discourse codes during one design activity.

\subsection{Contrasting Connections between Individuals}

A second consideration in comparing the conversation method and the moving stanza window method is that the conversation method suffers from the same limitation as many extant techniques for modelling CSCL (e.g., CORDTRA): it can model a group conversation, but it does not effectively model the participation of one individual in the context of a group discussion. The moving stanza window method, in contrast, can account for this important component of collaborative learning. 
(2017). In search of conversational grain size: Modelling semantic structure using moving stanza windows. Journal of Learning Analytics, 4(3), 123-139. http://dx.doi.org/10.18608/jla.2017.43.7

The reason for this difference is that the conversation method uses a single adjacency matrix to model each activity, and that matrix incorporates the contributions of all members of the group. There is thus no method for disentangling the contribution of any one individual. In contrast, the moving stanza window method models each utterance as an adjacency matrix, showing the connections one adjacency matrix (or one individual) contributes to the group discourse. As a result, we can use the moving stanza window method to examine the connections that each individual makes to the collaborative discussion of the group.

In this study, we modelled the contributions of two students, Jimmy and Connor, to the Hydraulic team's discussion. We constructed a network model of each of the two students' contributions, where each model included only those stanza windows in which the referring utterance belonged to that individual (Figure 5). These models thus represent the unique contributions to the team discussion made by each student.

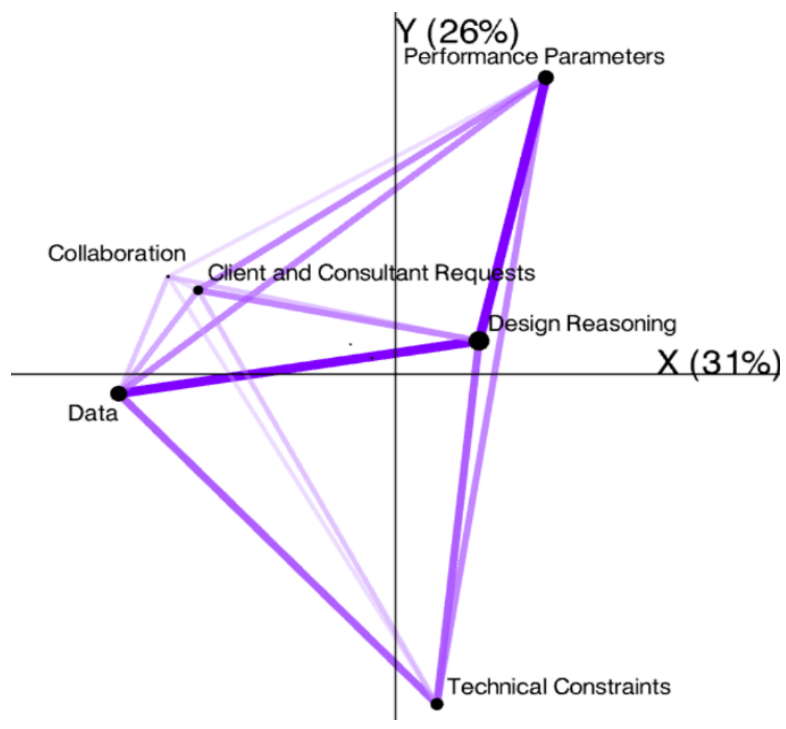

(a)

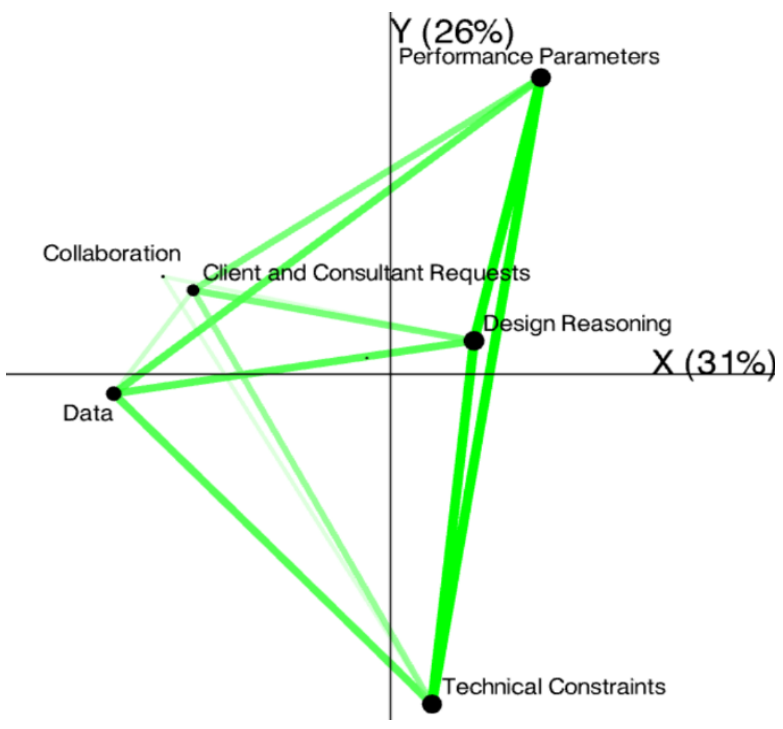

(b)

Figure 5. Moving stanza window model for Jimmy's (a) and Connor's (b) discourse. Thicker lines denote more frequent connections between discourse codes.

The networks using a moving stanza window method show that across all eleven activities or conversation, Connor's and Jimmy's individual contributions to the group discourse differ. This contrast is shown more clearly by computing the difference between the two individual network models (Figure 6). Figure 6 shows a higher number of connections in Connor's talk (green lines in the figure) between constraints and performance parameters, suggesting that Connor frequently made connections between the more technical attributes and inputs of the design problem. In contrast, Jimmy made more connections between data and design reasoning in the design discussion. 
(2017). In search of conversational grain size: Modelling semantic structure using moving stanza windows. Journal of Learning Analytics, 4(3), 123-139. http://dx.doi.org/10.18608/jla.2017.43.7

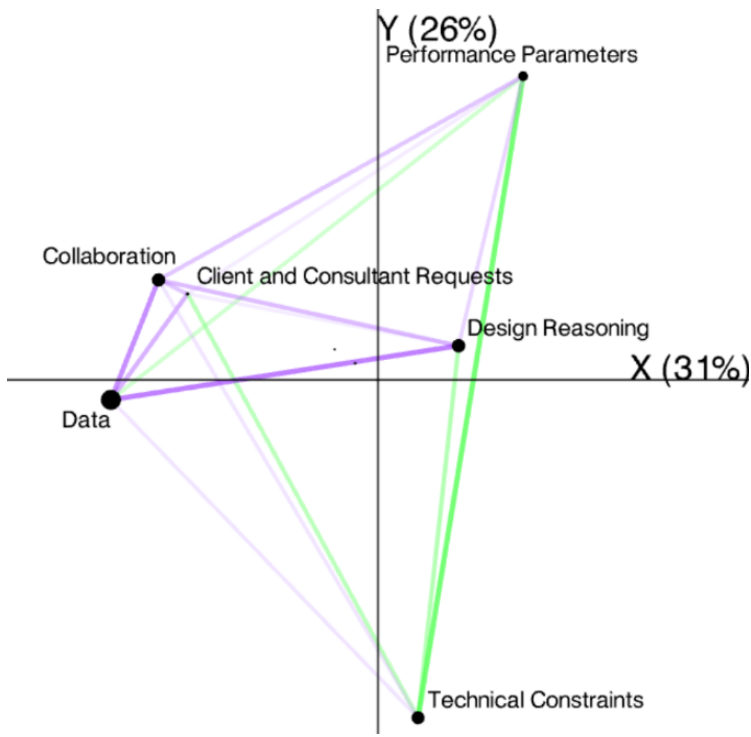

Figure 6. Subtracted network of Connor's and Jimmy's discourse, in which green connections occur more frequently in Connor's talk and purple connections occur more frequently in Jimmy's talk.

Table 3 illustrates this difference in a short excerpt from one of the group's discussions about interpreting experimental data. In this excerpt, Jimmy discussed design trade-offs and, in Jimmy's second comment (Line 2), he made a connection between data and design reasoning. He argued that graphs showed the results of benchmark testing (data) help the team make an "informed decision" (design reasoning) about their design choices. Two turns of talk later (Line 4), Connor added to the discussion by introducing information about specific attributes and inputs of the design: the performance parameters (payload, agility, and battery life) of some of the design choices that the team is considering (cadmium batteries and piezoelectric sensors), which connects to Jimmy's design reasoning comments.

Table 3. Brief Excerpt of the Hydraulic Team's Discussion of Findings during the Graphing Activity

Student Chat Utterance Code

1 Jimmy They all had both advantages and disadvantages. There was no "obvious" best choice.

Design Reasoning

2 Jimmy The graphs indicated the properties of all the different options and made a comparable visual illustration to make an informed decision on which combination to use.

Data, Design

Reasoning

3 Jordan The graphs detailed what aspects of power sources and control sensors are important - namely, the numerical data.

Data, Technical

Constraints

4 Connor I suggested using cadmium batteries with piezoelectric sensors; together they make a strong combination of payload and agility while keeping costs in a moderate range and having strong battery life.

Technical Constraints, Performance

Parameters, Data 
(2017). In search of conversational grain size: Modelling semantic structure using moving stanza windows. Journal of Learning Analytics, 4(3), 123-139. http://dx.doi.org/10.18608/jla.2017.43.7

This model using the moving stanza window method show that Connor builds on Jimmy's discussion about data and design reasoning by contributing information about technical constraints and performance parameters. The moving stanza window method separately modelled both Jimmy's original contributions to the team discussion and the fact that Connor's contribution built on Jimmy's utterance two lines before.

\section{DISCUSSION}

Our results suggest that the conversation method and the moving stanza window method identified different patterns of connection-making in student discourse. In particular, the conversation method summarized the connections made by student teams based on activity, but it did not identify individual contributions to team discussions. The moving stanza window method, in contrast, accounted for the connections that were made based on activity and temporal proximity; importantly, this method was also able to model the contributions of individual students to team conversations.

Of course, which of these models is the most appropriate depends on the theory of discourse being modelled and the assumptions of collaborative discourse. For example, if we assume that talk at the beginning of an activity frames everything that follows - or similarly, if talk at the end of an activity builds on everything that preceded it - then the conversation method is appropriate, because it models connections among all of the talk within a single activity. If, on the other hand, we assume that connections are sensitive to the temporal proximity of talk, then the moving stanza window method is a better choice, as this approach models connections locally within an activity such that very early turns of talk are not related to ideas that arise much later in the discussion.

An additional benefit of the moving stanza window method is that it also models the role of individual contributions to group discussions. By sliding a fixed number of lines across a dataset and defining a stanza for each line of chat, researchers can update the models of discourse after each chat. Therefore, moving stanza window ENA can make real time updates to the individual and group models of discourse each time a student chats in a virtual discussion. Many CSCL environments already include integrated feedback and assessment; however, the ability to model individual contributions to group discussions in a chat's recent temporal context would allow teachers the ability to assess real-time student performance in online environments (Shaffer, 2017).

In future work, the moving stanza window method could help researchers develop tools to support teacher use of learning analytic models within CSCL environments. Using this method, we could develop embedded assessments that automatically analyze student chat discourse to measure if students make certain connections between key elements during specific activities. By creating a predetermined set of core connections, we could create a network diagram of student learning that compares student and group connection-making with the target connections for that activity. Teachers could then use such models to monitor and support student achievement of learning outcomes as individuals and as teams. If students were not discussing key conceptual connections, the tool could suggest just-in-time 
(2017). In search of conversational grain size: Modelling semantic structure using moving stanza windows. Journal of Learning Analytics, 4(3), 123-139. http://dx.doi.org/10.18608/jla.2017.43.7

interventions that are specific, actionable, and based on student networks. Currently, we are developing a teacher interface tool that shows ENA models of student and group discussions in real time, allowing teachers to see what connections students make, or do not make, while engaging in our virtual internships (Shaffer, 2017).

This study, of course, is limited in that it focused on the activities of one group of students working in one CSCL context. The goal of this study was to provide an example of how two different segmentation techniques provided different models of discourse. By focusing on one team, we were able to go into richer detail about how an individual student contributed ideas in the context of other teammates' discussion. Of course, future analyses could dive deeper into the other groups in the sample or use the moving stanza window method on other data. Additionally, it is important to determine what sliding window size is most appropriate for different analyses (Graesser, Dowell, Clewley, \& Shaffer, in press) and we are investigating how to determine the appropriate window size that identifies the recent temporal context for a given learning environment (Shaffer, 2017).

However, this work empirically highlights a key theoretical distinction between models of connectivity in discourse, and perhaps more importantly, it demonstrates that the moving stanza window method makes it possible to use ENA to model both group discourse and the contributions of individuals to the group within a CSCL context.

\section{ACKNOWLEDGMENTS}

This work was funded in part by the National Science Foundation (DRL-0918409, DRL-0946372, DRL1247262, DRL-1418288, DRL-1661036, DRL-1713110, DUE-0919347, DUE-1225885, EEC-1232656, EEC1340402, REC-0347000), the MacArthur Foundation, the Spencer Foundation, the Wisconsin Alumni Research Foundation, and the Office of the Vice Chancellor for Research and Graduate Education at the University of Wisconsin - Madison. The opinions, findings, and conclusions do not reflect the views of the funding agencies, co-operating institutions, or other individuals.

\section{REFERENCES}

Arastoopour, G., Swiecki, Z., Chesler, N. C., \& Shaffer, D. W. (2015). Epistemic network analysis as a tool for engineering design assessment. Paper presented at the Annual Conference of the American Society for Engineering Education (ASEE 2015), 13 June, Seattle, WA, USA.

Arvaja, M., Salovaara, H., Häkkinen, P., \& Järvelä, S. (2007). Combining individual and group-level perspectives for studying collaborative knowledge construction in context. Learning and Instruction, 17(4), 448-459. http://dx.doi.org/10.1016/j.learninstruc.2007.04.003

Bakhtin, M. (1986). Speech genres and other late essays. Trans. Vern W. McGee. Austin, TX: University of Texas Press.

Bransford, J. D., Brown, A. L., \& Cocking, R. R. (1999). How people learn: Brain, mind, experience, and school. Washington, DC: National Academy Press. 
(2017). In search of conversational grain size: Modelling semantic structure using moving stanza windows. Journal of Learning Analytics, 4(3), 123-139. http://dx.doi.org/10.18608/jla.2017.43.7

Chi, M. T., Feltovich, P. J., \& Glaser, R. (1981). Categorization and representation of physics problems by experts and novices. Cognitive Science, 5(2), 121-152. http://dx.doi.org/10.1207/s15516709cog0502_2

Chesler, N. C., Ruis, A. R., Collier, W., Swiecki, Z., Arastoopour, G., \& Shaffer, D. W. (2015). A novel paradigm for engineering education: Virtual internships with individualized mentoring and assessment of engineering thinking. Journal of Biomechanical Engineering, 137(2), 024701. http://dx.doi.org/10.1115/1.4029235

Collier, W., Ruis, A. R., \& Shaffer, D. W. (2016). Local versus global connection making in discourse. In C. K. Looi, J. L. Polman, U. Cress, \& P. Reimann (Eds.) Transforming Learning, Empowering Learners: Proceedings of the 12th International Conference of the Learning Sciences (ICLS '16), 20-24 June 2016, Singapore (Vol. 1, pp. 426-433). International Society of the Learning Sciences. http://dx.doi.org/10.22318/icls2016.56

Cress, U., \& Hesse, F. W. (2013). Quantitative methods for studying small groups. In C. E. Hmelo-Silver, C. A. Chinn, C. K. K. Chan, \& A. O'Donnell (Eds.), The international handbook of collaborative learning (pp. 93-111). London: Routledge.

DiSessa, A. A. (1988). Knowledge in pieces. In G. Forman \& P. Pufall (Eds.), Constructivism in the computer age (pp. 47-70). Lawrence Erlbaum Publishers.

Dyke, G., Kumar, R., Ai, H., \& Rosé, C. P. (2012). Challenging assumptions: Using sliding window visualizations to reveal time-based irregularities in CSCL processes. In J. van Aalst, B. J. Reiser, C. Hmelo-Silver, \& K. Thompson (Eds.), The Future of Learning: Proceedings of the $10^{\text {th }}$ International Conference of the Learning Sciences (ICLS '12), 2-6 July 2012, Sydney, Australia (Vol. 1, pp. 363-370). International Society of the Leaning Sciences.

Gee, J. P. (1990). Social linguistics and literacies: Ideology in discourses. London: Falmer Press.

Gernsbacher, M. A. (1991). Cognitive processes and mechanisms in language comprehension: The structure building framework. In G. H. Bower (Ed.), The psychology of learning and motivation (pp. 217-263). Cambridge, MA: Academic Press. http://dx.doi.org/10.1016/S00797421(08)60125-5

Graesser, A. C., Dowell, N., Clewley, D., \& Shaffer, D. W. (in press). Agents in collaborative problem solving. International Journal of Computer-Supported Collaborative Learning.

Graesser, A. C., Gernsbacher, M. A., \& Goldman, S. R. (1997). Cognition. In T. A. van Dijk (Ed.), Discourse: A multidisciplinary introduction (pp. 292-319). Thousand Oaks, CA: Sage.

Hearst, M. A. (1994). Multi-paragraph segmentation of expository text. Proceedings of the $32^{\text {nd }}$ Annual Meeting of the Association for Computational Linguistics (ACL'94), 27-30 June 1994, Las Cruces, New Mexico, USA (pp. 9-16). Association for Computational Linguistics. Http://dx.doi.org/10.3115/981732.981734

Hmelo-Silver, C. E., Liu, L., \& Jordan, R. (2009). Visual representation of a multidimensional coding scheme for understanding technology-mediated learning about complex natural systems. Research and Practice in Technology Enhanced Learning, 4(3), 253-280. http://dx.doi.org/10.1142/S1793206809000714 
(2017). In search of conversational grain size: Modelling semantic structure using moving stanza windows. Journal of Learning Analytics, 4(3), 123-139. http://dx.doi.org/10.18608/jla.2017.43.7

Knight, S., Arastoopour, G., Shaffer, D. W., Shum, S. B., \& Littleton, K. (2014). Epistemic networks for epistemic commitments. In J. L. Polman, E. A. Kyza, D. K. O’Neill, I. Tabak, W. R. Penuel, A. S. Jurow, \& L. D'Amico (Eds.), Learning and Becoming in Practice: Proceedings of the International Conference of the Learning Sciences (ICLS '14), 23-27 June 2014, Boulder, CO, USA (Vol. 3, pp. 150-157). International Society of the Learning Sciences.

Landis, J. R., \& Koch, G. G. (1977). The measurement of observer agreement for categorical data. Biometrics, 33(1), 159-174.

Rosé, C., Wang, Y. C., Cui, Y., Arguello, J., Stegmann, K., Weinberger, A., \& Fischer, F. (2008). Analyzing collaborative learning processes automatically: Exploiting the advances of computational linguistics in computer-supported collaborative learning. International Journal of ComputerSupported Collaborative Learning, 3(3), 237-271.

Shaffer, D. W. (2012). Models of situated action: Computer games and the problem of transfer. In C. Steinkuehler, K. Squire, S. Barab (Eds.), Games learning, and society: Learning and meaning in the digital age (pp. 403-433). Cambridge, UK: Cambridge University Press.

Shaffer, D. W. (2014). User guide for epistemic network analysis web version 3.3. Madison, WI: Games and Professional Simulations Technical Report 2014-1.

Shaffer, D. W. (2017). Quantitative ethnography. Madison, WI: Cathcart Press.

Shaffer, D. W., Borden, F., Srinivasan, A., Saucerman, J., Arastoopour, G., Collier, W., Ruis, A. R., \& Frank, K. A. (2015). The $n$ Coder: A technique for improving the utility of inter-rater reliability statistics. Epistemic Games Group Working Paper 2015-01. University of Wisconsin-Madison.

Shaffer, D. W., Hatfield, D., Svarovsky, G., Nash, P., Nulty, A., Bagley, E. A., ... Mislevy, R. J. (2009). Epistemic network analysis: A prototype for $21^{\text {st }}$ century assessment of learning. The International Journal of Learning and Media, 1(1), 1-21.

Smagorinsky, P. (2011). Vygotsky and literacy research: A methodological framework (Vol. 2). Rotterdam, Netherlands/Boston, MA: Sense Publishers.

Stahl, G., Koschmann, T., \& Suthers, D. (2006). Computer-supported collaborative learning: An historical perspective. Cambridge handbook of the learning sciences (pp. 409-426). Cambridge, UK: Cambridge University Press.

Stahl, G. (2009). Studying virtual math teams. Springer Science and Business Media.

Suthers, D. D., \& Desiato, C. (2012). Exposing chat features through analysis of uptake between contributions. Proceedings of the $45^{\text {th }}$ Hawaii International Conference on System Sciences (HICSS-45), 4-7 January 2012, Maui, HI, USA (pp. 3368-3377). IEEE Computer Society. http://dx.doi.org/10.1109/HICSS.2012.274

Trausan-Matu, S., Dascalu, M., \& Rebedea, T. (2014). PolyCAFe-automatic support for the polyphonic analysis of CSCL chats. International Journal of Computer-Supported Collaborative Learning, 9(2), 127-156. http://dx.doi.org/10.1007/s11412-014-9190-y

Wells, G. (1999). Dialogic inquiry: Towards a sociocultural practice and theory of education. Cambridge, UK: Cambridge University Press. 